\title{
Effets des protéines laitières sur l'absorption du Fe, du Mg et du Zn mesurée par les variations temporelles de leurs teneurs dans l'aorte et la veine porte chez le rat
}

\author{
TO Pantako, M Passos, T Desrosiers, J Amiot \\ Université Laval, centre de recherches en sciences et technologie du lait, \\ département des sciences et technologie des aliments, Sainte-Foy (Quebec) Canada G1K 7P4
}

(Reçu le 31 mars 1992; accepté le 22 septembre 1992)

\begin{abstract}
Résumé - Cette étude visait à mettre en évidence la relation qui peut exister entre les différentes protéines du lait et de soja et l'absorption du fer $(\mathrm{Fe})$, du magnésium $(\mathrm{Mg})$ et du zinc $(\mathrm{Zn})$. À cet effet, des rats mâles de la lignée Sprague-Dawley ont eu, pendant $10 \mathrm{j}$, libre accès à 8 diètes protéiques entièrement équilibrées : lait, lactosérum, caséines, caséine $\alpha$, caséine $\beta$, $\alpha$-lactalbumine, $\beta$-lactoglobuline et soja. Au $10^{\mathrm{e}} \mathrm{j}$, des prélèvements de sang au niveau portal et aortique ont été faits sur des rats anesthésiés après $0,3,6$ et $12 \mathrm{~h}$ du début de la période de prise alimentaire maximale, afin d'évaluer l'absorption du $\mathrm{Fe}$, du $\mathrm{Mg}$ et du $\mathrm{Zn}$ par la méthode des différences porto-aortiques. Pour tous les groupes, on a noté un accroissement significatif et équivalent du poids corporel $(P<0,01)$, et une efficacité de conversion protéique comparable. Les teneurs en $\mathrm{Mg}$ et $\mathrm{Zn}$ ont été significativement affectées par les diètes protéiques $(P<0,05)$ aux 2 sites de prélèvement. Par contre, celles-ci n'avaient aucun effet significatif sur les teneurs en $\mathrm{Fe}$. $\dot{A}$ l'exception du $\mathrm{Mg}$, le temps d'échantillonnage influençait aussi significativement $(P<0,05)$ les teneurs des minéraux aux 2 sites. Des différentes porto-aortiques significativement différentes de zéro $(P<0,05)$, ont été observées à divers moments dans chaque groupe pour le $\mathrm{Mg}$ et le $\mathrm{Zn}$. On observe une certaine variabilité des résultats en ce qui concerne le maximum d'absorption. Cependant, le lactosérum et ses fractions semblent mieux stimuler l'absorption du $\mathrm{Zn}$, alors que la caséine $\beta$ démontre par rapport à toutes les sous-fractions du lait, une supériorité pour le $\mathrm{Mg}(P<$ $0,01)$.
\end{abstract}

absorption Fe-Mg-Zn / différence portoaortique / protéine laitière

Summary - Effect of dietary milk proteins on time-dependent variations in plasma $\mathrm{Fe}, \mathrm{Mg}$ and $\mathrm{Zn}$ levels in aorta and portal vein in rats. The effects of milk and soy dietary proteins on plas$\mathrm{ma} \mathrm{Fe}, \mathrm{Mg}$ and $\mathrm{Zn}$ levels in aorta and portal vein were investigated. Sprague-Dawley rats were fed ad libitum with equilibrated milk protein based diets during 10 days: milk, whey, caseins, $\alpha$-casein, $\beta$ casein, $\alpha$-lactalbumin, $\beta$-lactoglobulin and soy. On the 10th $d$, animals on each diet were anesthetized with halothane and blood samples were collected from aorta and portal vein $0,3,6$ and $12 \mathrm{~h}$ after the onset of the active eating period. $\mathrm{Fe}, \mathrm{Mg}$ and $\mathrm{Zn}$ were determined in these blood samples and porto-aortic differences were calculated. There was a significant and equivalent $(P<0.01)$ increase in body weight and comparable protein efficiency ratio (PER) in all groups. Whatever the site 
of sample collection, $\mathrm{Mg}$ and $\mathrm{Zn}$ concentrations were significantly $(\mathrm{P}<0.05)$ altered by protein diets. In contrast, there was no significant effect on Fe levels. In addition, except for $\mathrm{Mg}$, the mineral concentrations in both sites were significantly $(\mathrm{P}<0.05)$ affected by sampling time. Significant $(\mathrm{P}<0.05)$ porto-aortic differences higher than 0 were found in all groups for $\mathrm{Mg}$ and $\mathrm{Zn}$, but at varying sampling time, indicating that the kinetics of intestinal absorption were dependent on the protein fed. The maximum porto-aortic differences were variable among groups. Whey, $\alpha$-lactalbumin, and $\beta$-lactoglobulin fed groups exhibited the highest porto-aortic differences for $Z n$ compared with the other groups. However, $\beta$-casein seemed to enhance $\mathrm{Mg}$ absorption better than the other milk fractions $(\mathrm{P}<0.01)$.

\section{milk protein / plasma Fe-Mg-Zn / porto-aortic difference / rat}

\section{INTRODUCTION}

Pour le nourrisson, le lait constitue un aliment complet qui lui apporte tous les nutriments nécessaires à son développement. II continue d'être une bonne source de tous les nutriments à tous les âges de la vie, même si en fonction de l'âge et de la variété de la diète alimentaire, le lait et les produits laitiers ne continuent pas à contribuer de façon égale à l'apport nutritionnel recommandé en chacun des nutriments. C'est notamment le cas pour le $\mathrm{Mg}$, le $\mathrm{Fe}$ et le $\mathrm{Zn}, 3$ nutriments essentiels à la vie et dont la carence peut entraîner des problèmes d'anémie, de convulsion, d'obésité, de défense immunitaire, de croissance, etc (Kruse et al, 1932; Prasad, 1979; Park et al, 1986; Chen et al, 1988; Fairbanks et Beutler, 1988). Par ailleurs, le lait contribue aussi à fournir dans la diète des nutriments et autres facteurs pouvant améliorer significativement la biodisponibilité de certains nutriments. Selon Hallberg (1981) et Gordon et Godber (1989), I'acide ascorbique et les protéines d'origine animale seraient parmi les principaux éléments diététiques pouvant accroître significativement l'absorption du fer. Même si les protéines du lait, telle que l' $\alpha$-lactalbumine, ne semblent pas aussi efficaces que celles de la viande pour améliorer l'absorption du fer (Saxena et Subadra, 1988; Gordon et Godber, 1989). Kwock et al (1984) ont néanmoins suggéré que les caséines pourraient agir comme transporteur protéi- que du fer et ainsi faciliter l'absorption de ce minéral, ce qui pourrait contribuer à expliquer la bonne biodisponibilité du fer dans le lait de vache. Selon Hegenauer et al (1979), la capacité fixatrice du fer par les caséines serait même supérieure à celle de la lactoferrine et de la transferrine. Cet effet bénéfique des caséines sur l'absorption des minéraux, déjà mentionné par Maubois et Léonil (1989) en relation avec les alcalino-terreux et les oligo-éléments, serait dû à des segments peptidiques originaux des caséines : les phosphopeptides.

L'absorption du magnésium chez le rat se fait principalement au niveau du colon (Karbach, 1989; Hardwick et al, 1991), suivant un processus de diffusion dépendant de l'intensité et de la direction du flux aqueux à travers l'épithélium intestinal. Elle peut donc être favorisée par la présence de substances telles que $\mathrm{Na}$, le glucose ou l'urée (Lederer, 1983). Les travaux de Greger et al (1989) ont démontré que le lait et certains de ses sous-produits pouvaient également stimuler l'absorption du Mg. Ainsi, chez le rat, des rations à base de lait ou de lait avec lactose hydrolysé conduiraient à des concentrations de magnésium dans le sang et les tibias plus élevées qu'avec un régime à base de caséines. Ces résultats n'ont cependant pas été reproduits par Pointillart et al (1986) chez le porc avec des régimes à base de yaourt.

En ce qui concerne l'absorption du $\mathrm{Zn}$, Greger et al (1989) ont démontré chez le 
rat que des régimes à base de lait enrichi en lactose amélioraient de façon significative la teneur en zinc des os sans pourtant en modifier la teneur sérique. Même si selon Lonnerdal et al (1981) les caséines constituent les molécules de poids moléculaire élevé (Eckhert et al, 1977) comme facteur de liaison du $\mathrm{Zn}$ dans le lait de vache, ce serait davantage le lactose du lait qui serait responsable de l'effet positif enregistré. D'ailleurs, le sucrose, le lait avec lactose hydrolysé, ou les caséines n'auraient pas le même effet que le lactose (Wood et Hanssen, 1988; Greger et al, 1989) ou le glucose (Wapnir et al, 1989) sur l'absorption du $\mathrm{Zn}$. Par contre, il a aussi été rapporté que, chez l'humain, l'absorption du $\mathrm{Zn}$ peut être diminuée lorsque la teneur en protéines de l'alimentation est réduite ou que celles-ci proviennent de source végétale (Engel et al, 1966).

Les résultats parfois contradictoires publiés au sujet des effets des diètes sur l'absorption des différents minéraux considérés (Cousins et Smith, 1980) seraient en partie dus à des variations dans les conditions d'essais, dont la composition des diètes, mais aussi à des facteurs métaboliques dépendant du temps (cycles lumière-obscurité et/ou jeûne-prise alimentaire). Ainsi, Binnerts et al (1983) ont rapporté que le rythme d'absorption du Mg au cours de la journée était variable et dépendait du rythme alimentaire alors que le fer subissait une variation diurne, avec des valeurs matinales de $30 \%$ plus élevées que celles observées le soir (Fairbanks et Beutler, 1988). Dans cet ordre d'idées, et afin de tenir compte des capacités spatio-temporelles de compensation du tube digestif, Galibois et al (1987) ont suggéré, à la suite des travaux de Rérat et al $(1979,1985)$ sur l'absorption des protéines et des glucides, qu'une manière efficace de corréler les nutriments et en particulier les protéines avec la cinétique changeante de l'absorption intestinale des acides aminés, consistait à comparer les différences porto-aortiques de leurs concentrations dans le plasma en fonction du temps. Contrairement à d'autres, une telle approche conduit à des paramètres représentatifs de l'absorption dans la totalité du tube digestif. Cependant, un modèle beaucoup plus simplifié a été décrit par Jarousse et al (1980) et exploité par Bourdel et al (1981). Mieux adapté à l'étude de la cinétique intestinale d'absorption, il consiste en des prélèvements ponctuels de sang à la fois au niveau de la veine porte et de l'aorte abdominale. Les teneurs des minéraux dans les sérums collectés permettent de calculer les différences porto-aortiques et ainsi de comparer parallèlement les effets des différentes diètes sur la cinétique d'apparition de ceux-ci dans le sang porte.

Ce travail, selon le même point de vue, vise à vérifier de quelle manière les concentrations de $\mathrm{Fe}, \mathrm{Mg}$ et de $\mathrm{Zn}$ dans le flux sanguin se trouvent affectées par la présence de différentes protéines dans la diète. Utilisant le soja comme témoin, cette étude vise plus spécifiquement à évaluer chez le rat, l'effet comparatif des protéines du lait et de ses diverses fractions protéiques, lactosérum, caséines, caséine $\alpha$, caséine $\beta, \alpha$ lactalbumine, $\beta$-lactoglobuline, sur l'absorption de ces minéraux, en faisant appel aux différences porto-aortiques de leurs teneurs dans le plasma. De telles mesures faites à intervalles réguliers au cours de la période de prise alimentaire maximale, devraient permettre de corréler la cinétique d'absorption de $\mathrm{Fe}, \mathrm{Mg}$ et $\mathrm{Zn}$ avec la source protéique ingérée.

\section{MATÉRIEL ET MÉTHODES}

\section{Sources de protéines et composition des rations}

Les sources protéiques suivantes ont été utilisées : lait ( $87,3 \%$ de protéines), lactosérum 
(76\%), caséines $(91,4 \%)$, caséine $\alpha(91,7 \%)$, caséine $\beta(91,7 \%), \alpha$-lactalbumine $(90 \%), \beta$ lactoglobuline $(90 \%)$, et soja $(92 \%)$. Les protéines de lait ont été obtenues par ultrafiltration, diafiltration et concentration à partir de la poudre de lait écrémé Low Heat (coopérative fédérée du Québec, Qc, Canada) reconstitué et en utilisant des membranes organiques Romicon HF 26.5-43PM30 (Romicon Inc, Woburn, MA, USA) d'exclusion moléculaire moyenne de $30000 \mathrm{Da}$. Les caséines brutes ont été obtenues de la compagnie Sigma (Saint-Louis, MO, USA). La caséine $\alpha$ et la caséine $\beta$ ont été préparées d'après la méthode de Aschaffenburg (1963) et celle de Hipp et al (1952) par précipitation différentielle à l'urée, à partir des caséines entières produites par microfiltration et diafiltration de lait écrémé commercial à travers des membranes en alumine présentant $0,45 \mu \mathrm{m}$ de diamétre moyen de pores (Pantako, 1992). L' $\alpha$-lactalbumine et la $\beta$-lactoglobuline ont été obtenues de Nutrinov (Rennes, France), le lactosérum de Saputo (Saint-Hyacinthe, Québec, Canada) et les isolats de soja de Ralston Purina (Whitby, Ontario, Canada).

Ces sources protéiques ont été incorporées dans des rations ajustées pour contenir $20 \%$ de protéines, et équilibrées en ce qui concerne leur contenu en matières lipidiques, fibres, éléments minéraux, vitamines, lactose et amidon. Le soja a été supplémenté en L-méthionine $(0,1 \%)$. En outre, dans le cas de l' $\alpha$-lactalbumine le matériel brut protéique contenait une proportion réelle en cette protéine de $45 \%$, le reste étant constitué de $\beta$-lactoglobuline et de sérum albumine bovine. Finalement, le matériel brut protẻique de la ration $\beta$-lactoglobuline était pur à plus de $90 \%$. La composition de ces rations est présentée au tableau I.

\section{Expérimentation animale}

Deux cent cinquante-six rats mâles de la lignée Sprague-Dawley (Charles Rivers, SaintConstant, Québec) pesant entre 100 et $110 \mathrm{~g}$ ont été individuellement logés dans des cages suspendues faites de grillage en acier inoxydable. Les animaux ont d'abord été nourris pendant une semaine avec une moulée Charles Rivers RMH 4020 (Montréal Québec, Canada), afin de les adapter à un cycle inversé jour/nuit de $12 / 12 h$.
Puis les animaux ont été divisés en 8 groupes de 32 rats. À chaque groupe, a été assignè une des 8 diètes expérimentales auxquelles les rats ont eu libre accès ainsi qu'à l'eau pendant une période de $10 \mathrm{j}$. Au cours de cette période, les gains de poids et les prises alimentaires ont été enregistrès à 2 reprises pour tous les animaux afin de pouvoir calculer les coefficients d'efficacité protéique (CEP). Au $10^{\mathrm{e}}$ $j$, à 4 moments différents $(0,3,6$ et $12 \mathrm{~h})$ après le début de la période d'obscurité et de prise alimentaire maximale, 8 rats de chaque groupe expérimental ont été anesthésiés à l'halothane (4\%). La cavité abdominale a èté ouverte et le foie dégagé. La veine porte a été pincée à 0,5 $\mathrm{cm}$ du foie et environ $1,5 \mathrm{ml}$ de sang ont été prélevés à l'aide d'une seringue. La veine porte fut alors ligaturée à $1 \mathrm{~cm}$ du site de collecte précédent afin de prévenir le saignement. L'aorte au niveau abdominal a été dégagée des tissus et graisses environnants, et environ $1,5 \mathrm{ml}$ de sang ont été soutirés de la jonction iliacosacrale, à l'aide d'une seringue. Les sérums ont été recueillis immédiatement par centrifugation des échantillons de sang, et congelés à $-20^{\circ} \mathrm{C}$ pour analyses ultérieures.

\section{Analyses chimiques}

Le contenu en $\mathrm{Fe}, \mathrm{Mg}$ et $\mathrm{Zn}$ des ingrédients, des rations et des échantillons de sérum a été déterminé par spectrophotométrie d'absorption atomique (Perkin-Elmer 603, CT, USA). Le contenu azoté des sources de protéines a été évalué par la méthode Kjeldhal avec un appareil automatisé Kjelfoss 16210 (AVNN Foss Electrical, Danemark). La valeur énergétique des ingrédients et des rations a été obtenue à l'aide d'un calorimètre adiabatique automatique (Parr 1241 Adiabatic Calorimeter, Moline, IL, USA).

\section{Analyses statistiques}

Les effets de la source protéique, du gain de poids, de la prise alimentaire, du temps d'échantillonnage et du site de prélèvement du sang sur les teneurs en minéraux, ont été étudiés statistiquement par une analyse des variances avec le logiciel SAS (Freund et al, 1986). Le test de Duncan a été utilisé (avec SAS GLM) pour iden- 
Tableau I. Composition des régimes expérimentaux (\% p/p).

Composition of the experimental diets (\% w/w).

Ingrédients Soja Lait Caséines Caséine $\alpha$ Caséine $\beta$ Lactosérum $\alpha$-lactal $\beta$-lacto

\begin{tabular}{|c|c|c|c|c|c|c|c|c|}
\hline Soja & 21,8 & & & & & & & \\
\hline Lait & & 22,9 & & & & & & \\
\hline Caséines & & & 21,8 & & & & & \\
\hline Caséine $\alpha$ & & & & 21,8 & & & & \\
\hline Caséine $\beta$ & & & & & 21,9 & & & \\
\hline Lactosérum & & & & & & 26,3 & & \\
\hline$\alpha$-Lactalbumine & & & & & & & 21,5 & \\
\hline$\beta$-Lactoglobuline & & & & & & & & 22,3 \\
\hline Huile de maîs ${ }^{1}$ & 5,0 & 5,0 & 5,0 & 5,0 & 5,0 & 4,0 & 5,0 & 5,0 \\
\hline Fibres $^{2}$ & 5,0 & 5,0 & 5,0 & 5,0 & 5,0 & 5,0 & 5,0 & 5,0 \\
\hline Minéraux ${ }^{3}$ & 3,3 & 2,5 & 3,4 & 3,2 & 3,3 & 3,2 & 3,4 & 3,4 \\
\hline Vitamines 4 & 1,0 & 1,0 & 1,0 & 1,0 & 1,0 & 1,0 & 1,0 & 1,0 \\
\hline Choline bitartrate 5 & 0,2 & 0,2 & 0,2 & 0,2 & 0,2 & 0,2 & 0,2 & 0,2 \\
\hline L-Méthionine ${ }^{6}$ & 0,1 & & & & & & & \\
\hline Lactose ${ }^{7}$ & 2,4 & 2,4 & 2,4 & 2,4 & 2,4 & - & 1,9 & 1,9 \\
\hline Amidon de maïs ${ }^{8}$ & 61,3 & 61,0 & 61,1 & 61,4 & 61,2 & 60,3 & 62,0 & 61,2 \\
\hline $\begin{array}{l}\text { Énergie } \\
\text { (kcal/kg régime) }\end{array}$ & 4106,0 & 4143,2 & 4156,6 & 4255,1 & 4223,8 & 4239,0 & 4267,1 & 4212,4 \\
\hline
\end{tabular}

\footnotetext{
${ }^{1}$ Huile de maïs Mazola, Best Foods, Canada Starch (Ontario); ${ }^{2}$ Fibres Alphacel, ICN Biochemical (Cleveland, $\mathrm{OH}$, USA); ${ }^{3}$ Minéraux AIN 76 de ICN Biochemicals (Cleveland, OH, USA), véhiculés par la cellulose ( $\mathrm{mg} / \mathrm{kg}$ de régime) : $\mathrm{Ca}, 5$ 155; P, 3 983; Na, 1 020; K, 3 600; Cl, 1 570; Mg, 507; Mn, 53; Fe, 34,65; Zn, 31,5; I, 0,2; Se, 0,16; et Cr, 2. Éléments fournis par les sels suivants : $\mathrm{CaHPO}_{4}, \mathrm{NaCl}, \mathrm{K}_{3}$, citrate de fer $(16-17 \% \mathrm{Fe}), \mathrm{K}_{2} \mathrm{SO}_{4}, \mathrm{MgO}, \mathrm{MnCO}_{3}$, $\mathrm{ZnCO}_{3}, \mathrm{KIO}_{3}, \mathrm{Na}_{2} \mathrm{SeO}_{3} \cdot 5 \mathrm{H}_{2} \mathrm{O}$ et $\mathrm{CrK}\left(\mathrm{SO}_{4}\right)_{2} \cdot 12 \mathrm{H}_{2} \mathrm{O} .{ }^{4}$ Vitamines AIN $76 \mathrm{~A}$ de ICN Biochemicals (Cleveland, OH, USA), véhiculées par du sucrose ( $\mathrm{mg} / \mathrm{kg}$ de régime) : thiamine $\mathrm{HCl}, 6,0$; riboflavine, 6,0; pyridoxine $\mathrm{HCl}, 7,0$; acide nicotinique, 30; $\mathrm{D}$-calcium pantothenate, 16; acide folique, 2,0; cyanocobalamine (vit $\mathrm{B} 12$ ), 0,01 ; retinyl palmitate (vit A), 16,0; DL $\alpha$-tocophéryl acétate, 200,0; cholécalciferol (vit D3), 0,025; et ménaquinone (vit K), 0,05 ; ${ }^{5}$ Choline bitartrate, Sigma (St-Louis, MO, USA); ${ }^{6}$ L-Méthionine, Sigma (St-Louis, MO, USA); ${ }^{7}$ Lactose, Mallinckrodt (Kentucky, USA); ${ }^{8}$ Amidon de maïs, Best Foods, Canada Starch (Pointe-Claire, PQ).
}

tifier parmi les moyennes des variables dépendantes, celles qui avaient été significativement affectées par les traitements diététiques ou le moment d'échantillonage. Préalablement, une transformation logarithmique des données a été nécessaire pour rendre les variances des données homogènes selon le test de Bartlett (Snedecor et Cochran, 1980). À l'intérieur des groupes protéiques, une analyse des moindres carrés (SAS LSMEANS) a été effectuée sur les différences porto-aortiques afin de préciser à quels moments d'échantillonnage elles étaient significativement positives.

\section{RÉSULTATS ET DISCUSSION}

\section{Prise alimentaire, gain de poids, efficacité protéique}

La prise alimentaire totale a été significativement plus élevée $(P<0,05)$ dans le groupe nourri aux caséines que dans les autres groupes protéiques (tableau II). De plus, aucune différence significative n'a été obser- 
Tableau II. Effets des sources protéiques sur la prise alimentaire, le gain de poids et le CEP au cours d'une expérience de 10 jours *.

Effects of dietary protein sources on food intake, weight gain and PER during a 10 day experiment *.

\begin{tabular}{lcclll}
\hline Régimes & $\begin{array}{c}\text { Prise alimentaire } \\
(g)\end{array}$ & $\begin{array}{c}\text { Poids initial } \\
(g)\end{array}$ & $\begin{array}{c}\text { Poids final } \\
(g)\end{array}$ & $\begin{array}{c}\text { Gain de poids } \\
(g)\end{array}$ & $\begin{array}{c}\text { CEP } \\
(g)\end{array}$ \\
\hline Soja & $159,7 \pm 3,2^{\mathrm{bc}}$ & $144,4 \pm 1,02^{\mathrm{a}}$ & $205,4 \pm 2,6^{\mathrm{ab}}$ & $61,7 \pm 2,6^{\mathrm{abc}}$ & $1,92 \pm 0,06^{\mathrm{a}}$ \\
Lait & $163,4 \pm 2,0^{\mathrm{b}}$ & $143,4 \pm 0,72^{\mathrm{a}}$ & $208,0 \pm 1,8^{\mathrm{a}}$ & $63,5 \pm 1,2^{\mathrm{ab}}$ & $1,94 \pm 0,04^{\mathrm{a}}$ \\
Caséines & $170,6 \pm 2,5^{\mathrm{a}}$ & $142,9 \pm 1,07^{\mathrm{a}}$ & $209,4 \pm 2,0^{\mathrm{bcd}}$ & $66,0 \pm 1,8^{\mathrm{a}}$ & $1,93 \pm 0,04^{\mathrm{a}}$ \\
Caséine $\alpha$ & $145,5 \pm 2,5^{\mathrm{d}}$ & $143,2 \pm 0,96^{\mathrm{a}}$ & $199,7 \pm 2,0^{\mathrm{d}}$ & $56,5 \pm 1,7^{\mathrm{cd}}$ & $1,93 \pm 0,04^{\mathrm{a}}$ \\
Caséine $\beta$ & $151,9 \pm 4,4^{\mathrm{cd}}$ & $143,3 \pm 1,18^{\mathrm{a}}$ & $199,7 \pm 2,6^{\mathrm{d}}$ & $56,4 \pm 2,4^{\mathrm{d}}$ & $1,86 \pm 0,06^{\mathrm{a}}$ \\
Lactosérum & $159,2 \pm 2,6^{\mathrm{bc}}$ & $143,7 \pm 1,30^{\mathrm{a}}$ & $203,3 \pm 2,3^{\mathrm{abc}}$ & $60,4 \pm 2,1^{\mathrm{bcd}}$ & $1,90 \pm 0,05^{\mathrm{a}}$ \\
$\alpha$-lactalbumine & $158,0 \pm 2,8^{\mathrm{bc}}$ & $142,6 \pm 1,20^{\mathrm{a}}$ & $204,3 \pm 2,1^{\mathrm{abcd}}$ & $61,7 \pm 1,9^{\mathrm{abc}}$ & $1,95 \pm 0,04^{\mathrm{a}}$ \\
$\beta$-lactoglobuline & $153,0 \pm 2,2^{\mathrm{cd}}$ & $142,9 \pm 1,12^{\mathrm{a}}$ & $201,2 \pm 2,0^{\mathrm{cd}}$ & $58,3 \pm 1,2^{\mathrm{bcd}}$ & $1,90 \pm 0,04^{\mathrm{a}}$ \\
\hline
\end{tabular}

- Les valeurs représentent des moyennes de 32 rats \pm ES. Dans une colonne, les moyennes affichant les mêmes lettre ne sont pas significativement différentes $(P<0,05)$.

Values indicate means $\pm S E, n=32$ rats. Means in a column, with the same superscript letter are not significantly different $(\mathrm{P}<0.05)$.

vée entre le groupe nourri au soja et les groupes lait, caséine $\beta$, lactosérum et ses principales fractions protéiques. Dans tous les groupes, le poids corporel total s'est accru significativement $(P<0,006)$ durant les $10 \mathrm{j}$ de l'étude. Les résultats de gain de poids classent les sources protéiques de façon relativement équivalente au classement obtenu pour la prise alimentaire.

Aucune différence significative n'a été obtenue entre la majorité des sources de protéines laitières et le groupe soja. Par conséquent, les protéines du soja utilisées présentaient des qualités nutritionnelles comparables à celles reconnues aux protéines animales. Les quelques différences significatives de prise alimentaire et de gain de poids observées entre les sources protéiques étudiées n'ont pas permis de les distinguer sur le plan nutritionnel évalué en terme de coefficient d'efficacité protéique (CEP). Ce qui a permis de confirmer la validité du soja comme source protéique végétale de référence dans cette étude.
Les effets des diètes protéiques d'origines diverses sur l'utilisation des minéraux ont déjà été démontrés par des mesures globales telles que le gain de poids corporel (Moyer et al, 1978) ou par des mesures plus spécifiques comme les propriétés mécaniques de l'os (Crenshaw, 1986). Toutefois, une évaluation exhaustive des effets in vivo des diètes protéiques sur l'utilisation des minéraux nécessite de suivre des paramètres biologiques plus spécifiques, tels leur devenir métabolique par la mesure de leurs concentrations au niveau de l'aorte et de la veine porte, ainsi que leur absorption par le calcul des différences portoaortiques.

\section{Effets des diètes et du temps d'échantillonnage sur les teneurs sériques du $\mathrm{Fe}$, du $\mathrm{Mg}$ et du $\mathrm{Zn}$}

Les variations au niveau portal et aortique, des teneurs sériques de $\mathrm{Fe}, \mathrm{Mg}$ et de $\mathrm{Zn}$ 

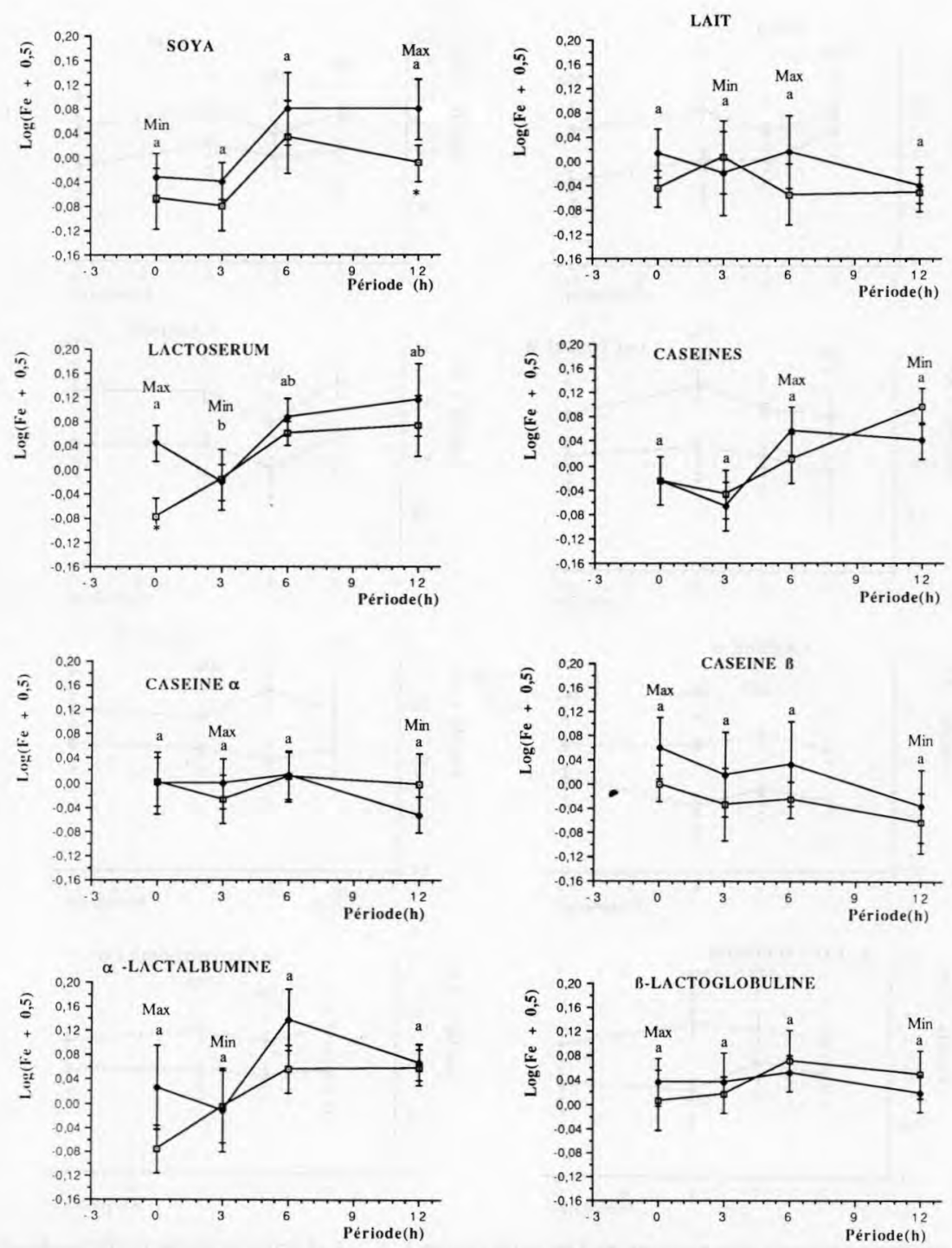

Fig 1. Variations sériques des teneurs de Fe dans l'aorte $(-\rightarrow)$ et dans la veine porte $(\rightarrow-)$. Les barres indiquent ET, $n=32$ rats/régime. Différence entre les teneurs portales et aortiques en une période donnée : ${ }^{*} P<0,05$. Les lettres indiquent l'amplitude des différences porto-aortiques. À l'intérieur du groupe, les points affichant les mêmes lettres ne sont pas significativement différents $(P<$ $0,05)$.

Plasma variations in aorta ( $\square-$ ) and portal $(\longrightarrow)$ Fe levels. Bars indicate SEM, $\mathrm{n}=32$ rats/diet. Difference between portal and arterial levels: ${ }^{*} P<0.05$. Letters indicate porto-aortic differences. Within the group, values with the same superscript letter are not significantly different $(P<0.05)$. 

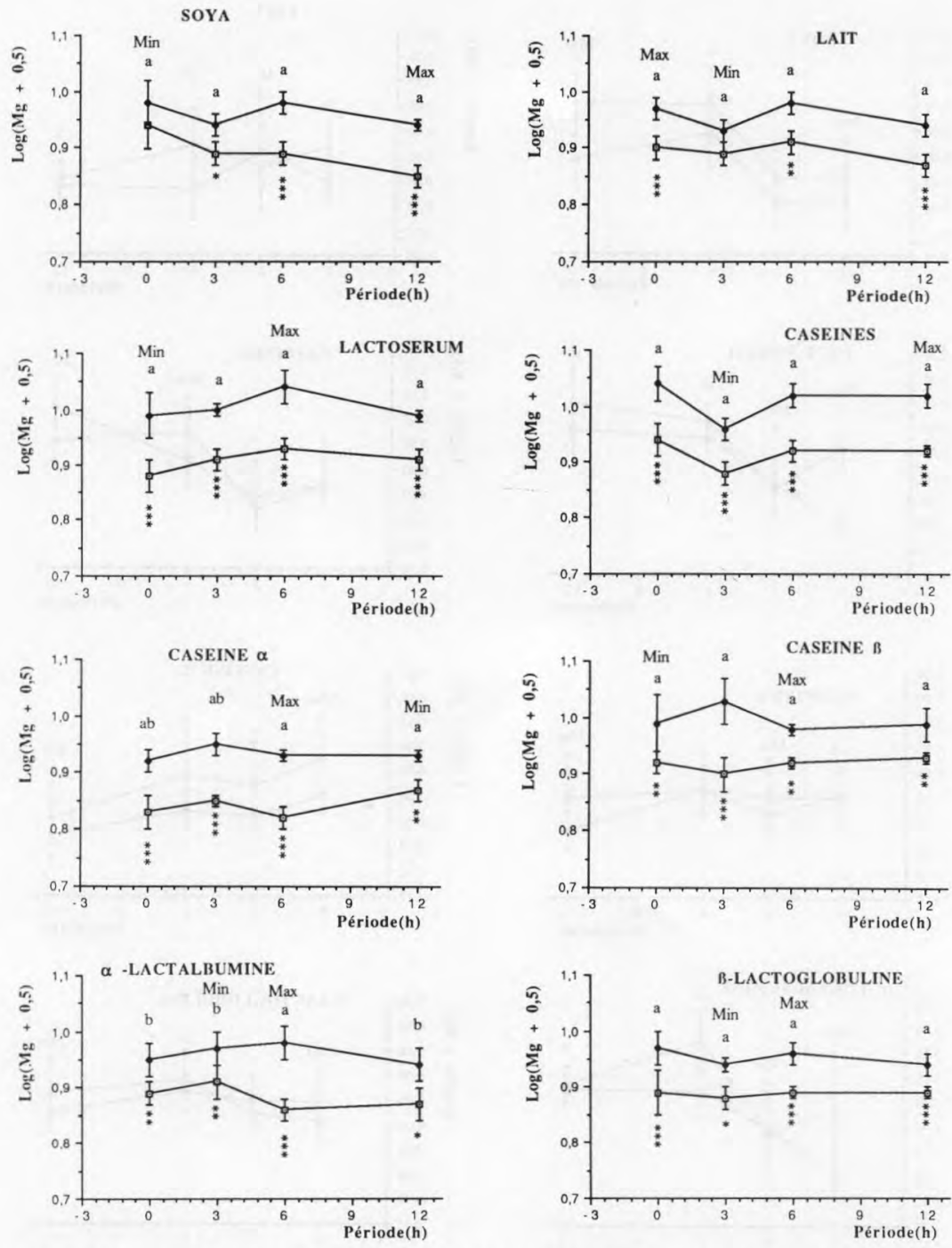

Fig 2. Variations sériques des teneurs de Mg dans l'aorte $(-\square-)$ et dans la veine porte $(-\rightarrow)$. Les barres indiquent $\mathrm{ET}, n=32$ rats/régime. Différence entre les teneurs portales et aortiques en une période donnée : ${ }^{\star} P<0,05,{ }^{\star *} P<0,005,{ }^{\star \star \star} P<0,0005$. Les lettres indiquent l'amplitude des différences porto-aortiques. À l'intérieur du groupe, les points affichant les mêmes lettres ne sont pas significativement différents $(P<0,05)$.

Plasma variation in aorta (- - ) and portal (- $\longrightarrow$ ) Mg levels. Bars indicate SEM, $\mathrm{n}=32$ rats/diet. Difference between portal and arterial levels: ${ }^{*} P<0.05,{ }^{\star \star} P<0.005,{ }^{\star * \star} P<0.0005$. Letters indicate porto-aortic differences. Within the group, values with the same superscript letter are not significantly different $(\mathrm{P}<0.05)$. 

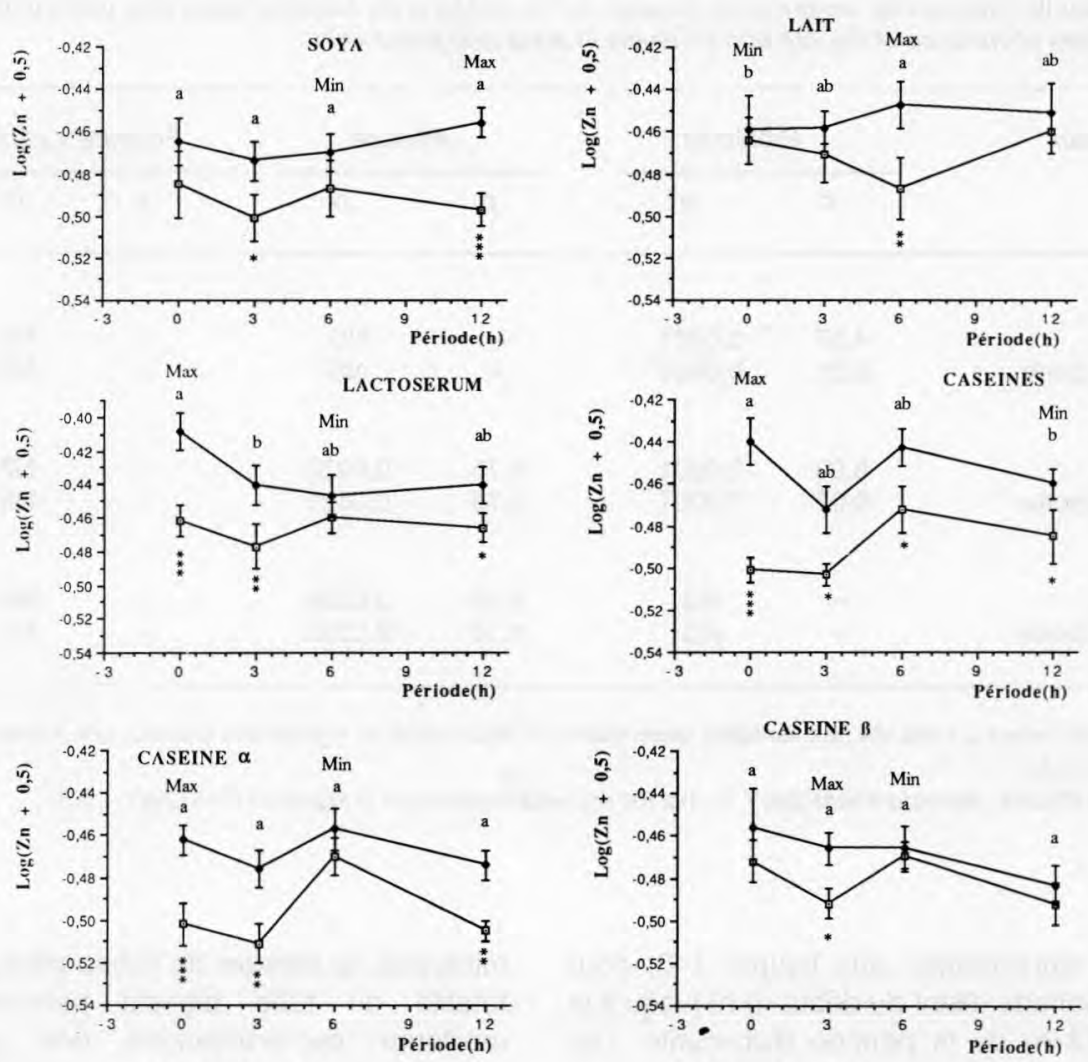

$\alpha$-LACTALBUMINE Max
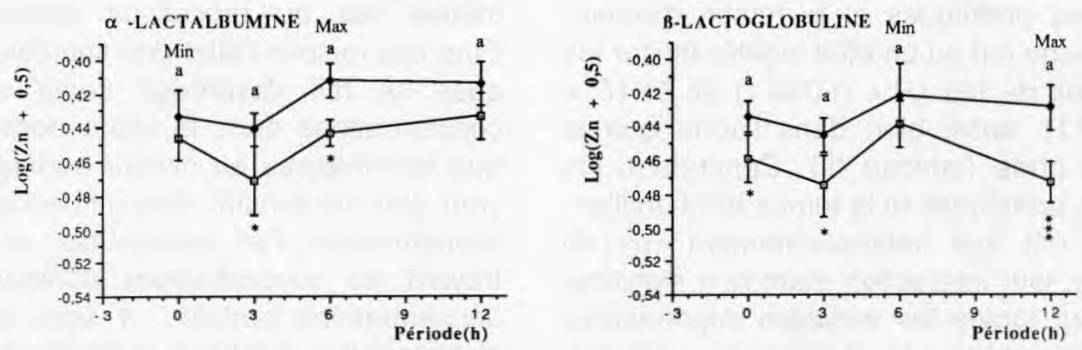

Fig 3. Variations sériques des teneurs de $\mathrm{Zn}$ dans l'aorte $(-\square)$ et dans la veine porte $(-\rightarrow)$. Les barres indiquent ET, $n=32$ rats/régime. Différence entre les teneurs portales et aortiques en une période donnée : ${ }^{*} P<0,05,{ }^{* *} P<0,005,{ }^{* *} P<0,0005$. Les lettres indiquent l'amplitude des différences porto-aortiques. À l'intérieur du groupe, les points affichant les mêmes lettres ne sont pas significativement différents $(P<0,05)$.

Plasma variations in portal (- - ) and aorta ( $\square-$ ) Zn levels. Bars indicate SEM, $n=32$ rats/diet. Difference between portal and arterial levels: ${ }^{*} P<0.05,{ }^{* *} P<0.005,{ }^{* * *} P<0.0005$. Letters indicate porto-aortic differences. Within the group, values with the same superscript letter are not significantly different $(\mathrm{P}<0.05)$. 
Tableau III. Analyses de variance des teneurs de $\mathrm{Fe}$, de $\mathrm{Mg}$ et de $\mathrm{Zn}$ dans l'aorte et la veine porte *. Analyses of variance of $\mathrm{Fe}, \mathrm{Mg}$ and $\mathrm{Zn}$ levels in aorta and portal vein *.

\begin{tabular}{|c|c|c|c|c|c|c|}
\hline \multirow[t]{2}{*}{ Minéraux } & \multicolumn{2}{|c|}{ Protéines } & \multicolumn{2}{|c|}{ Période } & \multicolumn{2}{|c|}{ Protéine $x$ période } \\
\hline & $F$ & $P$ & $F$ & $P$ & $F$ & $P$ \\
\hline \multicolumn{7}{|l|}{$M g$} \\
\hline Aorte & 4,57 & 0,0001 & - & NS & - & NS \\
\hline Veine porte & 5,31 & 0,0001 & - & NS & - & NS \\
\hline \multicolumn{7}{|l|}{$Z n$} \\
\hline Aorte & 8,93 & 0,0001 & 4,75 & 0,0032 & - & NS \\
\hline Veine porte & 9,02 & 0,0001 & 2,78 & 0,0421 & - & NS \\
\hline \multicolumn{7}{|l|}{$\mathrm{Fe}$} \\
\hline Aorte & - & NS & 3,26 & 0,0228 & - & NS \\
\hline Veine porte & - & NS & 3,16 & 0,0259 & - & NS \\
\hline
\end{tabular}

- Préalablement à I'ANOVA, les variables dépendantes $Y$ (exprimées en mg/100 ml) ont subi une transformation $\log (Y+0,5)$.

Before ANOVA, dependent variables $Y$ (in $\mathrm{mg} / 100 \mathrm{ml}$ ) were transformed in logarithm form $\log (\mathrm{Y}+0,5)$.

sont représentées aux figures $1-3$, pour une période allant du début $(0 \mathrm{~h})$ jusqu'à la fin $(12 \mathrm{~h})$ de la période d'obscurité. Les sources protéiques et le temps d'échantillonnage ont eu un effet significatif sur les niveaux de $\mathrm{Mg}(P<0,0001)$ et $\mathrm{Zn}(P<$ $0,0001)$, aussi bien dans l'aorte que la veine porte (tableau III). Cependant, les diètes protéiques et le temps d'échantillonnage ont agit indépendamment l'un de l'autre, leur interaction étant non significative sur toutes les variables dépendantes étudiées et pour les 3 éléments minéraux (tableau III).

Le sang prélevé au niveau portal n'ayant pas encore passé par le foie, il est possible de penser que les changements de concentrations en minéraux y soient principalement attribuables à l'apport alimentaire. Par ailleurs, les variations sanguines systémiques étant très tamponnées par la captation hépatique des minéraux, la mesure de l'absorption par la totalité du tube digestif permet une meilleure compréhension des phénomènes liés au processus d'absorption. Pour ces raisons l'effet des sources protéiques se fait davantage sentir sur les concentrations dans la veine porte, puisque les mesures au niveau aortique peuvent plus facilement être influencées par I'homéostasie. Par conséquent, en soustrayant les concentrations aortiques des concentrations portales, il sera possible d'éliminer les variations cycliques indépendantes des processus d'absorption reliés aux sources protéiques et/ou aux phénomènes physiologiques temporels impliqués.

\section{Fer}

Plus spécifiquement, la concentration de fer est généralement plus élevée dans la 
Tableau IV. Comparaison des sources protéiques selon leurs teneurs en minéraux dans l'aorte et la veine porte *.

Comparison of protein sources by mineral levels in aorta and portal vein *

Miné- Soja Lait Caséines Caséine $\alpha$ Caséine $\beta$ Lactosérum $\alpha$-Lactal $\beta$-Lacto
raux

\begin{tabular}{|c|c|c|c|c|c|c|c|}
\hline \multicolumn{8}{|l|}{ Aorte } \\
\hline $\mathrm{Fe}-0,02814^{a}$ & $-0,03678^{a}$ & $0,01662^{a}$ & $-0,00607^{a}$ & $-0,03065^{a}$ & $0,01276^{a}$ & $0,00893^{a}$ & $0,03516^{a}$ \\
\hline $\mathrm{Mg}^{*} 0,8885^{\mathrm{ab}}$ & $0,8915^{a b}$ & $0,9162^{a}$ & $0,8432^{c}$ & $0,9169^{a}$ & $0,9085^{\mathrm{ab}}$ & $0,8804^{b}$ & $0,8878^{\mathrm{ab}}$ \\
\hline $\mathrm{Zn}-0,4922^{\mathrm{d}}$ & $-0,47045^{b c}$ & $-0,49028^{d}$ & $-0,49697^{d}$ & $-0,48211^{\text {cd }}$ & $-0,46622^{b c}$ & $=-0,44834^{a}$ & $-0,46139$ \\
\hline \multicolumn{8}{|c|}{ Veine porte } \\
\hline $\mathrm{Fe} \quad 0,02335^{\mathrm{a}}$ & $-0,00970^{\mathrm{a}}$ & $0,00894^{a}$ & $-0,01222^{\mathrm{a}}$ & $0,01768^{a}$ & $0,06006^{\mathrm{a}}$ & $0,06209^{a}$ & $0,03539^{a}$ \\
\hline $\mathrm{Mg}^{*} 0,95949^{b}$ & 0,952270 & $1,01260^{\mathrm{a}}$ & $0,93276^{b}$ & $1,00168^{a}$ & $1,00809^{a}$ & $0,96063^{b}$ & $0,95187 b$ \\
\hline $\mathrm{Zn}-0,4664^{\mathrm{b}}$ & $-0,45421^{b}$ & $-0,45376^{b}$ & $-0,46726^{b}$ & $-0,46740^{b}$ & $-0,43410^{a}$ & $-0,42496^{a}$ & $-0,43253^{a}$ \\
\hline
\end{tabular}

Moyennes ( $n$ rats par groupe, $27<n<32$ ) exprimées en $\log (\mathrm{mg} / 100 \mathrm{ml}+0,5$ ). Dans une ligne, les moyennes affichant les mêmes lettres ne sont pas significativement différentes.

- Une analyse de contrastes "caséine $\beta$ vs (caséine $\alpha \alpha$-lactalbumine et $\beta$-lactoglobuline)", comparant l'effet de la caséine $\beta$ et des autres fractions protéiques du lait sur l'absorption du magnésium, a donné des résultats significatifs $(P<0,01)$.

Means $(27<n<32)$ are expressed in $\log (m g / 100 m l+0.5)$. Within a row, values with the same superscript letter are not significantly different.

" Contrast analysis: " $\beta$-casein vs ( $\alpha$-casein $\alpha$-lactalbumin and $\beta$-lactoglobulin)", gave significant results for $M g(\mathbb{P}<$ 0.01).

veine porte que dans l'aorte (tableau IV). Cependant, bien que les concentrations portales semblent globalement supérieures avec les diètes lactosérum et $\alpha$ lactalbumine (tableau IV), aucune diète ne réussit à se démarquer de façon significative des autres et ce, quel que soit l'instant de prélèvement considéré (tableau V-a). II est pourtant reconnu que l'apport de certains produits laitiers dans la diète peut avoir un effet favorable sur l'absorption du fer. Ainsi, Saxena et Subadra (1988) ont rapporté que la disponibilité du fer contenu dans les céréales peut être accrue par l'ajout de lait. Par contre, Gordon et Godber (1989) ont démontré que la viande de bœuf accroît jusqu'à 4 fois mieux que le lactosérum la biodisponibilité du fer contenu dans le riz, sans toutefois améliorer son absorption de façon notable. Mais au total, peu d'études exhaustives sur l'absorption du fer contenu dans des diètes ont été publiées, surtout à cause de la difficulté qu'il y a à mesurer efficacement sa biodisponibilité (Gordon et Godber, 1989). De la même manière dans la présente étude, les variations de concentration sanguine en fer enregistrées ont été trop faibles pour établir des distinctions statistiquement significatives sur cette base entre l'effet de différentes sources protéiques sur la biodisponibilité du fer.

Le lait ou le fromage contenant $165 \mathrm{mg}$ de calcium réduisent de 46 à $57 \%$ l'absorption du fer lorsque consommé avec des petits pains (Hallberg et al, 1991). Bien que le mécanisme par lequel le calcium interfère avec l'absorption du fer soit mal connu, Hallberg et al (1991) suggèrent que cette inhibition serait le résultat d'une com- 
Tableau V. Variations temporelles des niveaux sériques de $\mathrm{Fe}, \mathrm{Mg}, \mathrm{Zn}$ en fonction de la source protéique.

Time-dependent variations in plasma $\mathrm{Fe}, \mathrm{Mg}, \mathrm{Zn}$ levels by protein sources *

\begin{tabular}{|c|c|c|c|c|c|c|c|c|}
\hline \multirow[t]{2}{*}{ Protéines } & \multicolumn{4}{|c|}{ Aorte } & \multicolumn{4}{|c|}{ Veine porte } \\
\hline & $O h$ & $3 h$ & $6 h$ & $12 \mathrm{~h}$ & $O h$ & $3 h$ & $6 \mathrm{~h}$ & $12 h$ \\
\hline
\end{tabular}

$\mathrm{Fe}$

$\begin{array}{lr}\text { Soja } & -0,067^{\mathrm{a}} \\ \text { Lait } & -0,045^{\mathrm{a}} \\ \text { Caséines } & -0,026^{\mathrm{a}} \\ \text { Caséine } \alpha & 0,002^{\mathrm{a}} \\ \text { Caséine } \beta & 0,001^{\mathrm{a}} \\ \text { Lactosérum } & -0,077^{\mathrm{a}} \\ \alpha \text {-lactalbumine } & -0,078^{\mathrm{a}} \\ \beta \text {-lactoglobuline } & 0,006^{\mathrm{a}}\end{array}$

\section{$M g$}

\section{Soja}

Lait

Caséines

Caséine $\alpha$

Caséine $\beta$

Lactosérum

$0,939^{a}$

$0,895^{\mathrm{a}}$

$0,942^{a}$

$0,834^{\mathrm{b}}$

$0,925^{a b}$

$0,884^{a b}$

$\alpha$-lactalbumine $\quad 0,890^{\text {ab }}$

$\beta$-lactoglobuline

$-0,079^{a}$
$0,007^{a}$
$-0,048^{a}$
$-0,027^{a}$
$-0,033^{a}$
$-0,016^{a}$
$-0,005^{a}$
$0,016^{a}$

$-0,034^{a}$
$-0,055^{a}$
$0,011^{a}$
$0,009^{a}$
$-0,025^{a}$
$0,060^{a}$
$0,057^{a}$
$0,072^{a}$

$-0,010^{\mathrm{a}}$

$-0,052^{a}$

$0,097^{a}$

$-0,003^{a}$

$-0,063^{a}$

$0,073^{\mathrm{a}}$

$0,059^{\mathrm{a}}$

$0,050^{\mathrm{a}}$

$-0,033^{a}$
$0,013^{a}$
$-0,025^{a}$
$-0,001^{a}$
$0,063^{a}$
$0,044^{a}$
$0,026^{a}$
$0,037^{a}$

$-0,040^{\mathrm{a}}$

$-0,020^{a}$

$-0,068^{a}$

$-0,002^{a}$

$0,016^{a}$

$-0,021^{a}$

$-0,011^{a}$

$0,036^{a}$

$\begin{array}{rr}0,079^{\mathrm{a}} & 0,079^{\mathrm{a}} \\ 0,015^{\mathrm{a}} & -0,040^{\mathrm{a}} \\ 0,056^{\mathrm{a}} & 0,040^{\mathrm{a}} \\ 0,013^{\mathrm{a}} & -0,053^{\mathrm{a}} \\ 0,034^{\mathrm{a}} & -0,035^{\mathrm{a}} \\ 0,087^{\mathrm{a}} & 0,115^{\mathrm{a}} \\ 0,138^{\mathrm{a}} & 0,068^{\mathrm{a}} \\ 0,051^{\mathrm{a}} & 0,018^{\mathrm{a}}\end{array}$

$\begin{array}{ll}0,890^{a} & 0,886^{a c} \\ 0,893^{a} & 0,908^{a c} \\ 0,885^{a} & 0,917^{a c} \\ 0,848^{a} & 0,824^{b} \\ 0,899^{a} & 0,916^{a c} \\ 0,908^{a} & 0,931^{c} \\ 0,905^{a} & 0,859^{a b} \\ 0,884^{a} & 0,887^{a c}\end{array}$

$0,845^{a c}$
$0,869^{a b c}$
$0,916^{b c}$
$0,867^{a b c}$
$0,931^{b}$
$0,907 b c$
$0,877^{a b c}$
$0,885^{a b c}$

\section{$0,939 \mathrm{ac}$}

$0,927 \mathrm{ac}$

$0,964^{\text {ac }}$

$0,951^{\text {ac }}$

$1,028^{b}$

$1,005^{\mathrm{ab}}$

$0,975^{\mathrm{abc}}$

$0,941^{\mathrm{ac}}$

$\begin{array}{ll}0,978^{a c} & 0,944^{a} \\ 0,976^{a c} & 0,937^{a} \\ 1,019^{a c} & 1,021^{b} \\ 0,934^{b} & 0,928^{a} \\ 0,984^{a b c} & 0,994^{a b} \\ 1,044^{a c} & 0,992^{a b} \\ 0,981^{a b c} & 0,937^{a} \\ 0,960^{a b} & 0,941^{a}\end{array}$

Zn

$\begin{array}{lllllllll}\text { Soja } & -0,488^{\mathrm{abc}} & -0,501^{\mathrm{abc}} & -0,487^{\mathrm{ac}} & -0,497^{\mathrm{acd}} & -0,465^{\mathrm{a}} & -0,474^{\mathrm{a}} & -0,470^{\mathrm{ab}} & -0,456^{\mathrm{ab}} \\ \text { Lait } & -0,464^{\mathrm{ab}} & -0,471^{\mathrm{a}} & -0,487^{\mathrm{ac}} & -0,460^{\mathrm{bc}} & -0,459^{\mathrm{ab}} & -0,458^{\mathrm{ab}} & -0,449^{\mathrm{ab}} & -0,451^{\mathrm{ab}} \\ \text { Caséines } & -0,501^{\mathrm{ac}} & -0,503^{\mathrm{bc}} & -0,472^{\mathrm{ac}} & -0,485^{\mathrm{acd}} & -0,440^{\mathrm{ab}} & -0,472^{\mathrm{ab}} & -0,443^{\mathrm{abc}} & -0,460^{\mathrm{ab}} \\ \text { Caséine } \alpha & -0,502^{\mathrm{ac}} & -0,511^{\mathrm{bc}} & -0,470^{\mathrm{acd}} & -0,505^{\mathrm{acd}} & -0,462^{\mathrm{ab}} & -0,476^{\mathrm{a}} & -0,457^{\mathrm{ab}} & -0,474^{\mathrm{ab}} \\ \text { Caséine } \beta & -0,472^{\mathrm{abc}} & -0,492^{\mathrm{abc}} & -0,469^{\mathrm{acd}} & -0,492^{\mathrm{acd}} & -0,456^{\mathrm{ab}} & -0,466^{\mathrm{ab}} & -0,466^{\mathrm{ab}} & -0,483^{\mathrm{ab}} \\ \text { Lactosérum } & -0,462^{\mathrm{ab}} & -0,477^{\mathrm{ab}} & -0,460^{\mathrm{acd}} & -0,466^{\mathrm{ac}} & -0,408^{\mathrm{c}} & -0,441^{\mathrm{c}} & -0,447^{\mathrm{ab}} & -0,441^{\mathrm{ac}} \\ \alpha \text {-lactalbumine } & -0,446^{\mathrm{b}} & -0,471^{\mathrm{ab}} & -0,443^{\mathrm{bcd}} & -0,433^{\mathrm{b}} & -0,433^{\mathrm{bc}} & -0,441^{\mathrm{c}} & -0,412^{\mathrm{c}} & -0,414^{\mathrm{c}} \\ \beta \text {-lactoglobuline }-0,459^{\mathrm{ab}} & -0,475^{\mathrm{ab}} & -0,439^{\mathrm{bd}} & -0,472^{\mathrm{ac}} & -0,434^{\mathrm{abc}} & -0,445^{\mathrm{ab}} & -0,422^{\mathrm{bc}} & -0,429^{\mathrm{ac}}\end{array}$

\footnotetext{
- Moyennes des prélèvements effectués à chaque période sur 7-8 rats, exprimés en $\log (\mathrm{mg} / 100 \mathrm{ml}+0,5)$. Dans une colonne, les moyennes ayant le même exposant ne sont pas significativement différentes.

"Means ( $n=7-8 /$ sampling time) are expressed in $\log (\mathrm{mg} / 100 \mathrm{ml}+0,5)$. Within a column values with the same superscript letter do not significantly differ.
}

pétition entre les 2 éléments qui partageraient un site commun de fixation avec une ou plusieurs substances déterminantes au niveau des voies d'absorption. L'effet défavorable du calcium sur l'absorp- tion du fer (même à des doses normales dans une diète) présente des implications nutritionnelles importantes. Les teneurs relativement élevées en calcium $(0,5 \%)$ des diètes utilisées dans notre expérimentation 
(tableau 1), pourraient expliquer les résultats médiocres obtenus sur l'absorption du fer selon les résultats des concentrations portales et aortiques obtenues. Dans de telles conditions, l'effet inhibiteur du calcium est tel qu'il pourrait nuire à l'observation de l'effet spécifique des protéines sur les concentrations sériques de fer.

Toutefois, les profils temporels des différences porto-aortiques qui seraient de meilleurs indicateurs de l'absorption des nutriments, montrent des différences significatives pour 3 groupes protéiques : le soja $(P<0,05)$, le lactosérum $(P<0,03)$ et l' $\alpha$-lactalbumine $(P<0,03)$ (tableau $\mathrm{VI}$ ). Les maximums d'absorption atteints dès le début de la période de prise alimentaire maximale pour les groupes lactosérum et $\alpha$-lactalbumine et à la fin de cette période pour le groupe soja montrent bien l'effet significatif du temps d'échantillonage sur les teneurs en fer dans le sang portal $(P<$ 0,026 ) et aortique $(P<0,023)$ (tableau III). De plus, les différences porto-aortiques des groupes lactosérum et $\alpha$-lactalbumine au début de la période d'obscurité sont 4 fois plus élevées que celles du groupe soja et atteignent leur valeur minimale $3 \mathrm{~h}$ plus tard. En contre partie, pour le soja, le taux d'absorption du fer semble être à sa valeur minimale au début de la période de prise alimentaire. Le taux d'absorption progresse ensuite au cours de cette période et il est à sa valeur maximale après $12 \mathrm{~h}$.

Dans le cas des protéines du lactosérum et de l' $\alpha$-lactalbumine, on ne peut donc pas relier l'absorption du fer à la période de prise alimentaire (tableau VI) même si les taux sanguins enregistrés pendant cette période (tableau IV) semblent supérieurs à ceux obtenus pour les autres sources protéiques. L'effet des protéines de soja sur l'absorption du fer serait par ailleurs très lent puisque c'est le seul groupe pour lequel l'absorpton maximale est atteinte $12 \mathrm{~h}$ après le début de la période de prise alimentaire. Ces résultats pourraient donc contribuer à expliquer l'observation déjà faite que le soja puisse diminuer l'absorption du fer (Cook et al, 1981; Hallberg et Rossander, 1982). En contrepartie, les résultats obtenus ne permettent pas de confirmer que les caséines puissent avoir un rôle positif sur l'absorption du fer en agissant comme facteur de fixation et de transport du fer (Hegenauer et al, 1979; Kwock et al, 1984).

Bien que le modèle utilisé donne à penser que les diètes protéiques ont peu ou pas d'effet significatif sur les teneurs plasmatiques de fer (à cause sans doute de la teneur en calcium de nos diètes), il montre, en revanche, que la période d'échantillonnage influence significativement ces teneurs, rejoignant ainsi les observations de Fairbanks et Beutler (1988). En effet, alors que la saison, l'exercice et les repas normaux n'affectent pas les concentrations sériques de fer, ils notent par contre une variation diurne, avec des valeurs matinales de $30 \%$ plus élevées que celles observées le soir. Cette observation serait d'ailleurs confirmée ici par les taux maximaux d'absorption enregistrés pour la caséine $\beta$ et toutes les protéines du lactosérum, mais pas par les taux sanguins correspondants.

De manière générale, le fer est véhiculé dans la diète sous 2 formes différentes, chacun reliée à un mécanisme d'absorption spécifique (Hallberg, 1981). Ainsi, le fer hémique est absorbé dans les cellules de la muqueuse intestinale sous la forme d'un complexe fer-porphyrine à l'aide d'un récepteur spécifique (Gräsbäck et al, 1979). Le complexe porphyrique se brise à l'intérieur de ces cellules, probablement sous l'action d'oxygénases (Weintraub et al, 1968), et le fer ainsi libéré quitte cellesci sous la même forme que le fer nonhémique (Hallberg et Sölvell, 1967). La biodisponibilité du fer non-hémique est influencée par de nombreux facteurs diététiques, tels que, l'acide ascorbique, le phytate, les composés phénoliques et 
Tableau VI. Analyse aux moindres carrés des moyennes des différences porto-aortiques * Least means squares analysis of porto-aortic differences *.

\begin{tabular}{|c|c|c|c|c|c|c|}
\hline \multirow[t]{2}{*}{ Protéines } & \multicolumn{2}{|c|}{$M g$} & \multicolumn{2}{|c|}{$Z n$} & \multicolumn{2}{|c|}{$\mathrm{Fe}$} \\
\hline & Moy & $P^{* *}$ & Moy & $P^{\star *}$ & Moy & $P^{* *}$ \\
\hline \multicolumn{7}{|l|}{ Soja } \\
\hline $\mathrm{Oh}$ & $0,104^{\mathrm{m}}$ & 0,0619 & 0,012 & 0,0979 & $0,029^{m}$ & NS \\
\hline $3 \mathrm{~h}$ & 0,125 & 0,0172 & 0,016 & 0,0285 & 0,036 & NS \\
\hline $6 \mathrm{~h}$ & 0,234 & 0,001 & $0,010^{m}$ & NS & 0,049 & NS \\
\hline $12 \mathrm{~h}$ & $0,240^{\mathrm{M}}$ & 0,0001 & $0,025^{\mathrm{M}}$ & 0,0008 & $0,124^{\mathrm{M}}$ & 0,0558 \\
\hline \multicolumn{7}{|l|}{ Lait } \\
\hline $\mathrm{Oh}$ & $0,186^{M}$ & 0,004 & $0,003^{m}$ & NS & 0,060 & NS \\
\hline $3 \mathrm{~h}$ & $0,085^{m}$ & NS & 0,008 & NS & $-0,023^{m}$ & NS \\
\hline $6 \mathrm{~h}$ & 0,173 & 0,0011 & $0,024^{M}$ & 0,0015 & $0,076^{M}$ & NS \\
\hline $12 \mathrm{~h}$ & 0,169 & 0,0014 & 0,006 & NS & 0,011 & NS \\
\hline \multicolumn{7}{|l|}{ Caséines } \\
\hline $\mathrm{Oh}$ & 0,270 & 0,0001 & $0,039^{\mathrm{M}}$ & 0,0001 & 0,001 & NS \\
\hline $3 \mathrm{~h}$ & $0,200^{\mathrm{m}}$ & 0,0004 & 0,019 & 0,0116 & $-0,018$ & NS \\
\hline $6 \mathrm{~h}$ & 0,268 & 0,0001 & 0,019 & 0,0116 & $0,049^{\mathrm{M}}$ & NS \\
\hline $12 \mathrm{~h}$ & $0,280^{\mathrm{M}}$ & 0,0001 & $0,015^{m}$ & 0,0430 & $-0,061^{m}$ & NS \\
\hline \multicolumn{7}{|c|}{ Lactosérum } \\
\hline $\mathrm{Oh}$ & 0,269 & 0,0001 & $0,035^{\mathrm{M}}$ & 0,0001 & $0,118^{\mathrm{M}}$ & 0,0309 \\
\hline $3 \mathrm{~h}$ & 0,250 & 0,0001 & 0,023 & 0,0025 & $-0,008^{m}$ & NS \\
\hline $6 \mathrm{~h}$ & $0,306^{\mathrm{M}}$ & 0,0001 & $0,009^{m}$ & NS & 0,031 & NS \\
\hline $12 \mathrm{~h}$ & $0,220^{m}$ & 0,0001 & 0,016 & 0,0285 & 0,05 & NS \\
\hline \multicolumn{7}{|l|}{ Caséine $\alpha$} \\
\hline $\mathrm{Oh}$ & 0,200 & 0,0004 & $0,025^{M}$ & 0,0010 & 0,011 & NS \\
\hline $3 \mathrm{~h}$ & 0,256 & 0,0001 & 0,021 & 0,0043 & $0,025^{\mathrm{M}}$ & NS \\
\hline $6 \mathrm{~h}$ & $0,265^{\mathrm{M}}$ & 0,0001 & $0,009^{m}$ & NS & 0,001 & NS \\
\hline $12 \mathrm{~h}$ & $0,151^{m}$ & 0,0041 & 0,019 & 0,0116 & $-0,055^{m}$ & NS \\
\hline \multicolumn{7}{|l|}{ Caséine $\beta$} \\
\hline $\mathrm{Oh}$ & 0,197 & 0,0002 & 0,011 & NS & $0,069^{M}$ & NS \\
\hline $3 \mathrm{~h}$ & $0,345^{M}$ & 0,0001 & $0,016^{\mathrm{M}}$ & 0,0318 & 0,052 & NS \\
\hline $6 \mathrm{~h}$ & $0,176^{m}$ & 0,0081 & $0,002^{m}$ & NS & 0,065 & NS \\
\hline $12 \mathrm{~h}$ & 0,168 & 0,0057 & 0,006 & NS & $0,032^{m}$ & NS \\
\hline \multicolumn{7}{|c|}{$\alpha$-lactalbumine } \\
\hline $\mathrm{Oh}$ & 0,167 & 0,0016 & $0,009^{m}$ & NS & $0,114^{M}$ & 0,0268 \\
\hline $3 \mathrm{~h}$ & 0,178 & 0,0074 & 0,019 & 0,0116 & $-0,036^{m}$ & NS \\
\hline $6 \mathrm{~h}$ & $0,310^{\mathrm{M}}$ & 0,0001 & $0,020^{M}$ & 0,0072 & 0,090 & 0,0788 \\
\hline $12 \mathrm{~h}$ & $0,150^{\mathrm{m}}$ & 0,0044 & 0,013 & 0,0913 & 0,010 & NS \\
\hline \multicolumn{7}{|c|}{$\beta$-lactoglobuline } \\
\hline $\mathrm{Oh}$ & $0,190^{\mathrm{M}}$ & 0,0008 & 0,016 & 0,0327 & $0,028^{\mathrm{M}}$ & NS \\
\hline $3 \mathrm{~h}$ & $0,129^{m}$ & 0,0219 & 0,019 & 0,0116 & 0,026 & NS \\
\hline $6 \mathrm{~h}$ & 0,188 & 0,0004 & $0,011^{m}$ & NS & $-0,029$ & NS \\
\hline $12 \mathrm{~h}$ & 0,184 & 0,0011 & $0,028^{M}$ & 0,0002 & $-0,036^{m}$ & NS \\
\hline
\end{tabular}

- Moyenne des différences porto-aortiques $(\mathrm{mg} / 100 \mathrm{ml})$ mesurées à chaque instant de prélèvement sur 7-8 rats. ** Moyenne non nulle avec un niveau de signification $=P . \mathrm{m}$ Instant où la différence porto-aortique est minimale dans un groupe. $M$ Instant où la différence porto-aortique est maximale dans un groupe.

"Means of porto-aortic differences $(\mathrm{mg} / 100 \mathrm{ml})$ at each sampling time $(\mathrm{n}=7-8)$. ${ }^{* *}$ Non zero mean with $\mathrm{P}$ significance.

$m$ Time of minimum porto-aortic difference within a group. $M$ Time of maximum porto-aortic difference within a group. 
fréquemment la viande. On pense que la ferritine serait le médiateur de l'absorption de cette forme inorganique de fer à travers les cellules de la muqueuse intestinale (Fairbanks et Beutler, 1988). Par contre, ces facteurs, à l'exception de la viande, affectent peu l'absorption du fer hémique dans le tractus gastro-intestinal. II semble en fait que la source première du fer dans le système réticulum endothélial soit celui libéré par les érythrocytes parvenus au terme de leur vie (Fairbanks et Beutler, 1988 ), en vertu d'un processus de recyclage (phagocytose).

\section{Magnésium}

De la même façon que le fer, les concentrations en $\mathrm{Mg}$ au niveau portal sont systé- matiquement plus élevées que celles au niveau aortique, quel que soit du reste le temps de prélèvement (fig 2). Par contre, le temps d'échantillonnage ne semble pas affecter significativement les teneurs observées tant au niveau de l'aorte que de la veine porte (tableau III).

Il a été suggéré que le rythme d'absorption du magnésium au cours de la journée était variable et dépendait du rythme alimentaire (Shils, 1988). Löwick et al (1982) ont établi ainsi que l'absorption du Mg par heure est de 50 à $70 \%$ plus basse au cours de la matinée que de l'après-midi ou de la soirée. En dépit de cette observation, nos résultats montrent que le temps d'échantillonnage n'a aucun effet significatif sur les concentrations portales et aortiques de $\mathrm{Mg}$. En fait, une comparaison des

Tableau VII. Variations temporelles des différences porto-aortiques en fonction de la source protéique * Time-dependent variations in porto-aortic differences of mineral levels by protein sources *.

\section{Minéraux Soja Lait Caséines Caséine $\alpha$ Caséine $\beta$ Lactosérum $\alpha$-Lactal $\beta$-Lacto}

\begin{tabular}{|c|c|c|c|c|c|c|c|c|}
\hline \multicolumn{9}{|l|}{ Fer } \\
\hline $\mathrm{Oh}$ & $0,029^{a}$ & $0,060^{\mathrm{a}}$ & $0,001^{a}$ & $0,011^{a}$ & $0,069^{a}$ & $0,120^{a}$ & $0,114^{a}$ & $0,028^{a}$ \\
\hline $3 \mathrm{~h}$ & $0,036^{a}$ & $-0,023^{a}$ & $-0,018^{a}$ & $0,025^{a}$ & $0,052^{a}$ & $-0,008^{a}$ & $-0,036^{a}$ & $0,026^{a}$ \\
\hline $6 \mathrm{~h}$ & $0,049^{a}$ & $0,076^{a}$ & $0,049^{a}$ & $0,001^{a}$ & $0,065^{a}$ & $0,031^{a}$ & $0,090^{\mathrm{a}}$ & $-0,029^{a}$ \\
\hline $12 \mathrm{~h}$ & $0,124^{a}$ & $0,011^{\mathrm{ab}}$ & $-0,061^{b}$ & $-0,055^{b}$ & $0,032^{a b}$ & $0,050^{a b}$ & $0,010^{a b}$ & $-0,036^{a b}$ \\
\hline \multicolumn{9}{|c|}{ Magnésium } \\
\hline $\mathrm{Oh}$ & $0,104^{a}$ & $0,186^{a b}$ & $0,270^{\circ}$ & $0,200^{a b}$ & $0,197^{a b}$ & $0,269^{b}$ & $0,167^{a b}$ & $0,190^{\mathrm{ab}}$ \\
\hline $3 \mathrm{~h}$ & $0,125^{a}$ & $0,085^{a}$ & $0,200^{a b}$ & $0,256^{b}$ & $0,345^{b}$ & $0,250^{b}$ & $0,178^{a}$ & $0,129 a$ \\
\hline $6 \mathrm{~h}$ & $0,234^{a}$ & $0,172^{a}$ & $0,267^{\mathrm{a}}$ & $0,265^{a}$ & $0,176^{a}$ & $0,306^{a}$ & $0,310^{a}$ & $0,187^{a}$ \\
\hline $12 \mathrm{~h}$ & $0,240^{a}$ & $0,169^{a}$ & $0,280^{a}$ & $0,151^{a}$ & $0,168^{a}$ & $0,220^{a}$ & $0,150^{a}$ & $0,184^{a}$ \\
\hline \multicolumn{9}{|l|}{ Zinc } \\
\hline $\mathrm{Oh}$ & $0,012^{a b}$ & $0,003^{a b}$ & $0,039^{c}$ & $0,025^{\mathrm{ac}}$ & $0,011^{a b}$ & $0,035^{c}$ & $0,009^{a}$ & $0,016^{a b}$ \\
\hline $3 \mathrm{~h}$ & $0,016^{a}$ & $0,007^{a}$ & $0,019^{a}$ & $0,021^{a}$ & $0,016^{a}$ & $0,022^{a}$ & $0,019^{a}$ & $0,019^{a}$ \\
\hline $6 \mathrm{~h}$ & $0,010^{a}$ & $0,024^{a}$ & $0,019^{a}$ & $0,009^{a}$ & $0,002^{a}$ & $0,009^{a}$ & $0,020^{\mathrm{a}}$ & $0,011^{a}$ \\
\hline $12 \mathrm{~h}$ & $0,025^{a b}$ & $0,006^{a}$ & $0,015^{a b}$ & $0,019^{a b}$ & $0,006^{a}$ & $0,016^{a b}$ & $0,012^{a b}$ & $0,027^{b}$ \\
\hline
\end{tabular}

- Moyennes des prélèvements effectués à chaque période sur 7 ou 8 rats $(\mathrm{mg} / 100 \mathrm{ml})$. Dans une ligne, les moyennes affichant les mêmes lettres ne sont pas significativement différentes (LSMEANS: $P<0,05$ ).

Means ( $\mathrm{n}=7$ or $8 /$ sampling time) are expressed in ( $\mathrm{mg} / 100 \mathrm{ml})$. Within a row, values with the same superscript letter are not significantly different (LSMEANS: $\mathrm{P}<0.05$ ). 
niveaux sériques en fonction des sources protéiques période par période, ne permet pas non plus de tirer des conclusions claires, quel que soit le site de prélèvement considéré (tableau V-b). Bien que les valeurs moyennes sur $12 \mathrm{~h}$ dénotent des différences significatives entre les sources protéiques (tableau IV), on ne peut pas attribuer ces spécificités à un moment particulier au cours de la période de prise alimentaire. II est certes vrai que les rats mangeant ad libitum, on ne peut pas parler de rythme alimentaire. Par conséquent, le taux d'absorption associé à chaque source protéique serait peu modifié durant la période d'étude concernée, spécialement au cours des 2 dernières périodes (6 et $12 \mathrm{~h}$ ) où les différentes protéines présentent pratiquement la même absorption (tableau VII).

De manière générale, pour toutes les sources protéiques, les teneurs en $\mathrm{Mg}$ dans l'aorte sont significativement supérieures $(P<0,05)$ à celles obtenues pour la caséine $\alpha$ (tableau IV). Par ailleurs, les concentrations de ce minéral dans la veine porte sont significativement supérieures $(P<0,05)$ pour les groupes caséines, caséine $\beta$ et lactosérum, comparativement aux autres groupes (tableau IV). Une analyse de contrastes, faite sur les résultats des concentrations aortiques et portales a permis de montrer que, la caséine $\beta$ est, de toutes les fractions protéiques élémentaires du lait, caséine $\alpha$, caséine $\beta, \alpha$ lactalbumine et $\beta$-lactoglobuline, celle qui stimulerait le plus efficacement l'absorption du magnésium $(P<0,01)$.

Cependant, quelle que soit la source protéique, la parallélisme notable en ce qui concerne l'évolution des courbes des teneurs portales et aortiques du $\mathrm{Mg}$ en fonction du temps (fig 2), démontre bien que ces phénomènes ne peuvent être considérés indépendamment l'un de l'autre. Tel qu'antérieurement démontré par Rérat et al $(1979,1985)$ et Galibois et
Savoie (1987), le calcul des différences porto-aortiques constituerait dans ce contexte un meilleur moyen pour évaluer l'effet de différents régimes alimentaires sur l'absorption des nutriments. Ainsi, les différences porto-aortiques les plus élevées sont obtenues avec le groupe caséine $\beta 3 \mathrm{~h}$ après le début de la période de prise alimentaire maximale (tableau VI), ce qui confirme les résultats déjà notés au niveau des concentrations portales et aortiques. Des taux maximaux d'absorption inférieurs à celui obtenu pour la caséine $\beta$, sont enregistrés pour la caséine $\alpha$, le lactosérum et l' $\alpha$-lactalbumine après $6 \mathrm{~h}$, et après $12 \mathrm{~h}$ pour les groupes soja et caséines. Enfin, pour les groupes lait et $\beta$ lactoglobuline, il est impossible de relier l'absorption du Mg à la période de prise alimentaire maximale puisque dans les 2 cas, le maximum d'absorption est atteint au moment où débute cette période.

Selon ces résultats, les différences porto-aortiques révèlent une cinétique d'absorption différente entre les sources protéiques. Ainsi, pour le groupe soja, l'absorption du $\mathrm{Mg}$ augmente régulièrement pendant toute la période de prise alimentaire maximale et semble atteindre une valeur maximale après $12 \mathrm{~h}$, comparativement à des sources protéiques comme la caséine $\beta$, le lactosérum et la caséine $\alpha$, pour lesquels le maximum d'absorption est atteint après $3-6 \mathrm{~h}$. Ce phénomène pourrait s'expliquer par le double mécanisme de transport intestinal du $\mathrm{Mg}$ : le premier, transcellulaire et saturable, utilise des transporteurs; le second, paracellulaire, procède par simple diffusion à de fortes concentrations intraluminales (Shils, 1988). Ainsi, les protéines laitières dont la digestion est plus rapide, font vraisemblablement davantage appel au premier mécanisme que les protéines du soja, car les caractéristiques structurales et la spécificité des enzymes digestives entraînent pour le soja une digestion plus lente qui accroit 
leur temps de résidence dans le petit intestin et la libération plus lente de petits peptides et d'acides aminés absorbables (Yudkin, 1977; Amiot et al, 1981). Par conséquent, dans le cas des protéines de soja, l'absorption des acides aminés se fait plus lentement et plus loin dans lintestin que pour les protéines laitières, ce qui peut conduire à une déficience en acides aminés nécessaires entre autres pour la synthèse in situ des transporteurs impliques dans l'absorption active du $\mathrm{Mg}$.

De nombreux auteurs ont rapporté que l'apport de $\mathrm{Ca}$ en grandes quantités dans la diète pouvait réduire significativement l'absorption du Mg. Chez des adultes bien portants s'alimentant avec des diètes pauvres en $\mathrm{Ca}(300 \mathrm{mg} / \mathrm{j})$ et riche en $\mathrm{Ca}$ (2 $000 \mathrm{mg} / \mathrm{j})$, Norman et al (1981) ont observé après 4 semaines, une baisse significative de l'absorption du $\mathrm{Mg}$ dans l'iléon des sujets soumis à la diète riche en $\mathrm{Ca}$. L'effet négatif du Ca sur l'absorption du Mg pourrait s'expliquer par une compétition au niveau d'un système de transport commun, une modification de la perméabilité membranaire au $\mathrm{Mg}$ induite par le $\mathrm{Ca}$, et une modulation d'un transporteur spécifique de $\mathrm{Mg}$ par le $\mathrm{Ca}$ (Hardwick et al, 1991). De plus, Brink et al (1992) ont observé chez des rats que la supplémentation en $\mathrm{Ca}$ diminuait l'absorption du $\mathrm{Mg}$, mais uniquement pour une ration riche en $P$ ce qui s'expliquerait par la formation de complexe insoluble ( $\mathrm{Ca}, \mathrm{Mg}$, Phosphate). Néanmoins, dans les conditions expérimentales de la présente étude, contrairement au fer, nous n'avons observé aucune interaction négative entre la teneur en $\mathrm{Ca}$ des diètes et l'absorption du Mg.

\section{Zinc}

Tout comme pour $\mathrm{Fe}$ et $\mathrm{Mg}$ la concentration de $\mathrm{Zn}$ est plus élevée dans la veine porte que dans l'aorte (fig 3 ) pour toutes les sources protéiques et quel que soit l'instant d'échantillonnage. Les effets de $l ' \alpha$-lactalbumine sur les teneurs en $\mathrm{Zn}$ dans l'aorte sont significativement supérieurs $(P<0,05)$ à ceux observés avec les autres sources protéiques relativement équivalentes entre elles à l'exception des caséines, de la caséine $\alpha$, de la caséine $\beta$ et du soja qui présentent des valeurs significativement inférieures (tableau IV). En revanche, au niveau portal, le lactosérum et ses fractions protéiques, l' $\alpha$-lactalbumine, la $\beta$-lactoglobuline, ont des effets comparables et significativement supérieurs, comparativement aux autres sources protéiques (tableau IV).

A l'exception de la $\beta$-lactoglobuline, les sources de protéines laitières dont le lait, l' $\alpha$-lactalbumine et la caséine $\beta$ atteignent un taux maximal d'absorption mesurée par le calcul des différences porto-aortiques, plus rapidement que le soja au cours de la période de prise alimentaire (tableau VI). Par contre, pour les caséines, le lactosérum et la caséine $\alpha$, il ne semble pas y avoir de relation directe entre la période de prise alimentaire maximale et l'absorption du $\mathrm{Zn}$. Ces résultats peuvent donc contribuer à confirmer que les sources de protéines végétales, telles le soja, ne permettent par une bonne absorption du zinc.

En effet, les protéines de plus grandes dimensions telles que les caséines et le soja présentent dans l'ensemble une moins bonne aptitude à transporter le $\mathrm{Zn}$ (Lederer, 1985). De même, dans le lait de fermme le $\mathrm{Zn}$ se déplace surtout avec des protéines dont les molécules sont de petites dimensions alors que dans le lait de vache il est fixé sur les grosses molécules de caséine qui le libèrent difficilement; cette disparité entre les poids moléculaires des ligands (Cousins et Smith, 1980), pourrait peut-être expliquer la très grande différence de biodisponibilité du $\mathrm{Zn}$ notée entre ces 2 sortes de lait. Dans ce sens, l' $\alpha$-lactalbumine, qui est parmi les composantes majeures du lait celle qui a la plus 
faible masse moléculaire, semble être la protéine stimulant le mieux l'absorption du zinc. Selon Kitts (1990), cette protéine jouerait un rôle biologique encore plus important chez l'humain. L'effet bénéfique de l' $\alpha$-lactalbumine sur l'absorption du $\mathrm{Zn}$ s'expliquerait d'ailleurs par le fait que cette protéine possède un site spécifique pour fixer le cation $\mathrm{Zn}^{2+}$ (Murakami et Berliner, 1983; Baumy et Brulé, 1988).

Forbes et al (1984) ont rapporté que le Ca pouvait négativement affecter l'absorption du $\mathrm{Zn}$, principalement en présence d'acide phytique. Castillo-Duran (1991) ont montré que $300 \mathrm{mg}$ de $\mathrm{Ca}$ dans un verre de lait inhibait l'assimilation du zinc, et les conclusions de leurs travaux tendent à confirmer l'opinion répandue selon laquelle le lait et les produits seraient défavorables à l'utilisation de cet élément par l'homme. Par contre, Spencer et al (1984) n'ont pu confirmer cet effet inhibiteur du $\mathrm{Ca}$ sur l'absorption du $\mathrm{Zn}$. II en va de même pour la présente étude, où la présence de $\mathrm{Ca}$ dans les diètes ne semble pas avoir affecté négativement les teneurs sériques de zinc ni son absorption.

\section{CONCLUSION}

De manière globale, il apparaît que les diètes protéiques ont beaucoup plus d'effet sur l'absorption du Mg que sur celle du $\mathrm{Fe}$ et du $\mathrm{Zn}$. De plus, il ressort que dans le lait, l' $\alpha$-lactalbumine est le principal facteur protéique associé à la biodisponibilité du $\mathrm{Zn}$ et du $\mathrm{Fe}$, alors que la caséine $\beta$ favorise davantage celle du $\mathrm{Mg}$. Une étude adéquate de la cinétique d'absorption des nutriments associée aux diètes protéiques à digestion lente, nécessiterait cependant des mesures porto-aortiques à de nombreux instants, et, surtout, pendant un intervalle d'observation suffisamment long (Galibois et Savoie, 1987). En effet, on observe que dans certains cas l'absorp- tion n'est pas complète après $12 \mathrm{~h}$. En outre, une analyse plus exhaustive des effets de diètes protéiques sur l'utilisation des 3 minéraux par l'organisme devrait tenir compte de l'impact de ces diètes sur leur solubilisation réelle dans l'intestin, laquelle semble être un bon indicateur de leur biodisponibilité potentielle (Wien et Schwartz, 1985). Cette étude est présentement en cours.

\section{RÉFÉRENCES}

Amiot J, Brisson GJ, Delisle J, Goulet G (1981) Nitrogenous products released by in vitro enzymatic hydrolysis of food proteins. Nutr Rep Int 24, 513-529

Aschaffenburg R (1963) Preparation of $\beta$-casein by a modified urea fractionation method. J Dairy Res 30, 259-260

Baumy JJ, Brulé G (1988) Binding of bivalent cations to $\alpha$-lactalbumin and $\beta$-lactoglobulin: effect of $\mathrm{pH}$ and ionic strength. Lait $68,33-48$

Binnerts WT, Lôwick MRH, Groot EH (1983) On the importance of magnesium in drinking water in trace elements. In: Analytical chemistry in medicine and biology. Vol II (Bräter P, Schramel P, eds) Walter de Gruyter and cie, New York, NY, USA

Bourdel G, Kande J, Robin D, Robin P (1981) Quantitative and qualitative circadian variations of amino acid intestinal efflux in mixed-fed and in protein-meal-fed rats. J Nutr 111, 1528-1535

Brinck EJ, Beynen AC, Dekker PR, Van Beresteijn CH, Van der Meer R (1992) Interaction of calcium and phosphate decreases ileal magnesium solubility and apparent magnesium absorption in rats. J Nutr $122,580-586$

Castillo-Duran C (1991) Studies on the bioavailability of zinc in humans. IX. Interaction of beef-zinc with iron, calcium and lactose. Nutr Res 11, 429-438

Chen MD, Lin PY, Lin WH, Cheng V (1988) Zinc in hair and serum of obese individuals in Taiwan. Am J Clin Nutr 48, 1307-1309

Cook JD, Morck TA, Lynch SR (1981) The inhibitory effect of soy products on non-heme iron absorption in man. Am J Clin Nutr 34, 2622-2629 
Cousins AJ, Smith KI (1980) Zinc binding properties of bovine and human milk in vitro - influence of changes in zinc content. Am J Clin Nutr 33, 1083-1087

Crenshaw TD (1986) Reliability of dietary Ca and $P$ levels and bone mineral content as predictors of bone mechanical properties at various time periods in growing swine. $J$ Nutr $116,2155-2170$

Eckhert C, Sloan M, Duncan JR, Hurley LS (1977) Zinc binding: a difference between human and bovine milk. Science 195, 789 790

Engel RW, Miller RF, Price NO (1966) Metabolic patterns in pre-adolescent children: XIII. Zinc balance. In: Zinc metabolism (Prasad AS, ed) Charles C Thomas, Springfield, IL, 326-338

Fairbanks F, Beutler E (1988) Iron. In: Modern nutrition in health and disease (Shils ME et Young VR, eds). Lea et Febiger, Philadelphia, PA, 193-226

Forbes RM, Parker HM, Erdman JW Jr (1984) Effects of dietary phytate, calcium and magnesium levels on the zinc bioavailability to rats. J Nutr 114, 1421-1425

Freund RJ, Littell RC, Spector PC (1986) SAS system for linear models. SAS Institute Inc, Cary, NC, USA

Galibois I, Savoie L (1987) Relationship between amino acid intestinal effluent in rat and in vitro protein digestion products. Nutr Res 7, 65-79

Galibois I, Parent G, Savoie L (1987) Effect of dietary proteins on time-dependent changes in plasma amino acid levels and on liver protein synthesis in rats. J Nutr 117, 2027-2035

Gordon DT, Godber JS (1989) The enhancement of non-heme iron bioavailability by beef protein in rat. J Nutr $119,446-452$

Gräsbäck R, Kouvonen I, Lundberg M, Tenhunen R (1979) An intestinal receptor for heme. Scand J Haematol 23, 59

Greger JL, Gutkowski CM, Khazen RR (1989) Interactions of lactose with calcium, magnesium and zinc in rats. J Nutr 119, 1691-1697

Hallberg $L$ (1981) Bioavailability of dietary iron in man. Ann Rev Nutr 1, 123-147

Hallberg L, Sölvell L (1967) Absorption of hemoglobin iron in man. Acta Med Scand 181, 335-354
Hallberg L, Rossander L (1982) Effect of soy protein on non-heme iron absorption in man. Am J Clin Nutr 36, 514-520

Hallberg L, Brune M, Erlandsson M, Sandberg AS, Sossander-Hultén L (1991) Calcium: effect of different amounts of non-heme- and heme-iron absorption in humans. Am J Clin Nutr 53, 112-119

Hardwick LL, Jones MR, Brautbar N, Lee DBN (1991) Magnesium absorption: mechanisms and influence of vitamin D, calcium and phosphate. J Nutr 121, 13-23

Hegenauer J, Saltman P, Ludwig D, Ripley L, Ley L (1979) Iron-supplemented cow milk. Identification and spectral properties of iron bound to casein. J Agric Food Chem 27, 1294-1301

Hipp NJ, Groves ML, Custer JH, McMeekin TL (1952) Separation of $\alpha-\beta$ - and $\gamma$-casein. J Dairy Sci 35, 272-281

Jarousse C, Lardeux R, Bourdel G, GirardGloba A, Rosselin G (1980) Portal insulin and glucagon in rats fed proteins as a meal: immediate variations and circadian modulations. I Nutr 110, 1764-1773

Karbach U (1989) Cellular-mediated and diffusive magnesium transport across the descending colon of the rat. Gastroenterology 96 , 1282-1289

Kitts DD (1990) Biodisponibilité du calcium laitier : rôle des phosphopeptides de la caséine. Nutr Actual 14, 25-38

Kwock RO, Keen CL, Hegenauer J, Saltman P, Hurley LS, Lonnerdal B (1984) Retention and distribution of iron added to cow's milk and human milk as various salts and chelates. $J$ Nutr 114, 1454-1461

Kruse HD, Orent ER, Mc Collum EV (1932) Studies on magnesium deficiency in animals. 1. Symptomatology resulting from magnesium deprivation. J Biol Chem 96, 519-529

Lederer J (1983) Magnésium : Mythes et réalités. Maloine Editeurs, Paris, France

Lederer J (1985) Le zinc en pathologie et en biologie. Maloine éditeurs, Paris, France

Lonnerdal B, Keen CL, Hurley LS (1981) Iron, copper, zinc, and manganese in milk. Ann Rev Nutr 1, 149-174

Löwick MRH, Groot EH, Binnerts WT (1982) Magnesium and public health: XVI. Univ Missou$r i$ (Delbert D, ed) Hemphill Publishing Co, MO, USA 
Maubois JL, Léonil J (1989) Peptides du lait à activité biologique. Lait 69, 245-269

Moyer GL, Wilson HD, Schedl HP (1978) Effects of dietary protein on body weight, mucosal growth, and calcium absorption in the rat. Dig Dis 23, 545-549

Murakami K, Berliner LJ (1983) A distinct zinc binding site in the $\alpha$-lactalbumins regulates calcium binding. Is there a physiological role for this control? Biochemistry 22, 3370-3374

Norman DA, Fordtran JS, Brinkley LJ, Zenwekh JE, Nicar MH, Strowig SM, Pak CYC (1981) Jejunal and ileal adaptation to alterations in dietary calcium: changes in calcium and magnesium absorption and pathogenic role of parathyroid hormone and 1,25dihydroxyvitamin D. J Clin Invest 67, 15991603

Pantako OT (1992) Contribution à l'étude des effets des protéines laitières sur les processus de digestion et d'absorption du calcium, fer, magnésium, phosphore et zinc. Thèse de doctorat, Université Laval, Québec, Canada

Park JHY, Grandjean CJ, Antonson DL, Vanderhoof JA (1986) Effects of isolated zinc deficiency on the composition of skeletal muscle, liver and bone during growth in rats. J Nutr 116, 610-617

Pointillart A, Cayron B, Guéguen L (1986) Utilisation du calcium et phosphore et minéralisation osseuse chez le porc consommant du yaourt. Sci Aliments 6, 15-30

Prasad AS (1979) Zinc in human nutrition. CRC Press, Boca Raton, FL, USA

Rérat A, Vaissade P, Vaugelade P (1979) Absorption kinetics of amino acid and reducing sugars during digestion of barley or wheat meals in the pig: preliminary data. Ann Biol Anim Biochim Biophys 19, 739-747

Rérat A, Simoes-Nunes C, Lacroix M, Vaugelade P, Vaissade P (1985) Comparative quantitative intestinal absorption of aminoacid mixtures of the same composition offered either in the free form or as an enzymatic hydrolysate of milk proteins to conscious pigs. CR Séances Acad Sci Paris 300 (ser III), 293-296

Saxena A, Subadra S (1988) The effect of whole milk, milk protein and some constituent amino acids on the in vitro availability of iron from cereal milks. Nutr Res 8, 717-724

Shils ME (1988) Magnesium. In: Modern nutrition in health and disease (Shils ME, Young VR, eds). Lea and Febiger, Philadelphia, PA, 159-192

Snedecor GW, Cochran WG (1980) Statistical methods. lowa State Univ Press, Ames, IA, 7 th edn

Spencer H, Kramer L, Norris C, Osis D (1984) Effect of calcium and phosphorus on zinc metabolism in man. Am J Clin Nutr 40, 12131218

Wapnir RA, Stiel L, Shih-Yu L (1989) Zinc intestinal absorption : effect of carbohydrates. Nutr Res 9, 1277-1284

Weintraub LR, Weinstein MR, Huser HJ, Rafal S (1968) Absorption of hemoglobin iron. The role of a heme-splitting substance in intestinal mucosa. J Clin Invest 47, 531-539

Wien EM, Schwartz R (1985) Dietary calcium exchangeability and bioavailability. Evaluation and potential uses of an in vitro digestion procedure. In: Nutritional bioavailability of calcium (Kies C, ed) Am Chem Soc, Washington, DC, 1-16

Wood RJ, Hanssen DA (1988) Effect of milk and lactose on zinc absorption in lactoseintolerant postmenopausal women. $J$ Nutr $118,982-986$

Yudkin J (1977) Recherches nutritionnelles effectives sous les auspices de la Fédération internationale de laiterie. Bull Annu, Doc No 94, IDF, Bruxelles, Belgique 